Document downloaded from:

http://hdl.handle.net/10251/48059

This paper must be cited as:

Sencadas, VJGDS.; Areias Cepa, A.; Areias, A.; Botelho, G.; Fonseca, A.; Neves, I.; Gómez Ribelles, JL.... (2012). Determination of the parameters affecting electrospun chitosan fiber size distribution and morphology. Carbohydrate Polymers. 87(2):1295-1301.

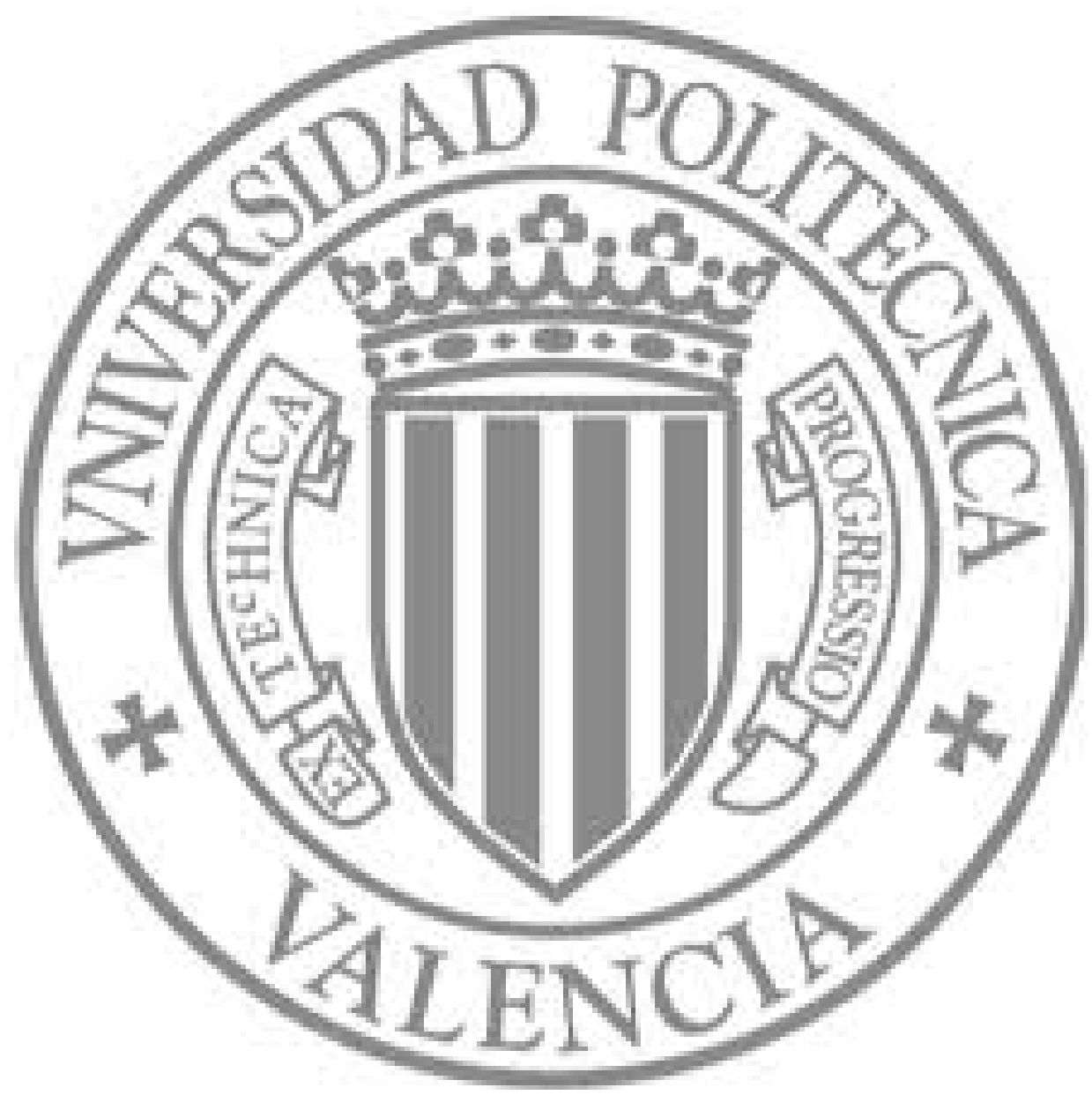

The final publication is available at

http://dx.doi.org/10.1016/j.carbpol.2011.09.017

Copyright Elsevier 


\section{Determination of the parameters affecting electrospun chitosan fiber \\ 2 size distribution and morphology}

3

4 V. Sencadas ${ }^{1}$; D. M. Correia ${ }^{2}$; A. Areias ${ }^{1}$; G. Botelho ${ }^{2}$; A. M. Fonseca ${ }^{2}$; I. C. Neves ${ }^{2}$; J.

5 L. Gómez-Ribelles ${ }^{3,4,5}$ and S. Lanceros-Mendez ${ }^{1}$

6

$7{ }^{1}$ Centro/Departamento de Física, Universidade do Minho, Campus de Gualtar, 47108058 Braga, Portugal

$9{ }^{2}$ Dept. Química, Centro de Química, Universidade do Minho, Campus de Gualtar, 10 4710-057 Braga, Portugal;

$11{ }^{3}$ Centro de Biomateriales e Ingeniería Tisular, Universidad Politécnica de Valencia, 12 Camino de Ver s/n, 46022 Valencia, Spain

${ }^{4}$ Centro de Investigación Príncipe Felipe, Autopista del Saler 16, 46013 Valencia,

14 Spain

*e-mail: vsencadas@fisica.uminho.pt

\section{Abstract}

19 The production of chitosan nanofiber mats by electrospinning presents serious difficulties due to the lack of suitable solvents and the strong influence of processing 
parameters on the fiber properties. Two are the main problems to be solved: to control the properties of the solution in order to obtain large area uniform fiber mats by having a stable flow rate and to avoid sparks during the process, damaging the fiber mats. In this work chitosan electrospun mats have been prepared form solutions of trifluoroacetic acid / dichloromethane mixtures, allowing to solve the aforementioned problems. Mats with uniform fibers of submicron diameters without beads were obtained. Further, the influence of the different solution and process parameters on the mean fiber diameter and on the width of the distribution of the fiber sizes has been assessed. Solvent composition, needle diameter, applied voltage and traveling distance were the parameters considered in this study.

\section{Introduction}

Increasing attention has been given in recent years to natural polymers, such as polysaccharides, due to their abundance in nature, unique structures and characteristics with respect to synthetic polymers (Honarkar \& Barikani, 2009). Chitosan is a natural linear polysaccharide composed of glucosamine and $\mathrm{N}$-acetyl glucosamine units linked by $\beta$ (1-4) glycosidic bonds. Although naturally present in some microorganisms and fungi, commercial chitosan is industrially produced by partial deacetylation of chitin by removal of acetamide groups. Chitin is the second most abundant natural polysaccharide after cellulose. It is mainly found in crustacean shells (shrimp, crab, etc.), insect cuticle and cell walls of fungi (Baldrick, 2010; Fernandez-Megia, NovoaCarballal, Quiñoá \& Riguera, 2005; Krajewska, 2005; Malafaya, Silva \& Reis, 2007; Ravi Kumar, 2000). The degree of deacetylation, DD, which defines the distinction between chitin and chitosan, is not precisely established. 
The term chitosan is found in the literature to describe polymers of chitosan with different molecular weights, viscosity and degree of deacetylation (40-98\%) (Baldrick, 2010). However, the term chitosan is generally applied when the degree of deacetylation is above $70 \%$ and the term chitin is used when the degree of deacetylation is below 20\% (Baldrick, 2010).

Chitosan in its crystalline form is usually insoluble in aqueous solutions above a $\mathrm{pH}$ of 7. However, due to the existence of primary amine groups, the structure can be protonated and the protonate free amine groups on glucosamine facilitate the solubility of the molecule, being therefore highly soluble in acid pH (Pillai, Paul \& Sharma, 2009; Yaghobi \& Hormozi, 2010).

Chitosan offers many structural possibilities for chemical modifications in order to induce novel properties, functions and applications, in particular in the biomedical area. It is nontoxic, biocompatible and biodegradable and therefore an excellent material for biomedical applications (Jayakumar, Menon, Manzoor, Nair \& Tamura, 2010; Jayakumar, Prabaharan, Nair, Tokura, Tamura \& Selvamurugan, 2010; Jayakumar, Prabaharan, Sudheesh Kumar, Nair \& Tamura, 2011).

Recently, much attention has been paid to chitosan based nanofibers as biomaterial. There are several methods for the fabrication of nanofibers, making use of chemical, thermal and electrostatic principles (Beachley \& Wen, 2010). The polymer nanofiber fabrication methods most commonly associated with biomedical applications are electrospinning, self-assembling, peptide reactions and phase separation. The electrospinning process has attracted much attention for the production of polymer fibers as it can produce them with diameters in the range from several micrometers down to tens of nanometers, depending on the polymer and processing conditions (Jayakumar, Prabaharan, Nair \& Tamura). The nanofibers produced by this technique 
are formed from a liquid polymer solution or melt that is feed through a capillary tube into a region of an electric field generated by connecting a high voltage power source to the capillary tube.

Chitosan nanofibers were successfully prepared by Ohkawa et al. (Ohkawa, Cha, Kim, Nishida \& Yamamoto, 2004). The authors studied the solvent effect on the morphology of electrospun chitosan nanofibers by varying chitosan relative concentration with different solvents. The solvents tested were diluted hydrochloric acid, acetic acid, formic acid and trifluoracetic acid (TFA). It was found that when the concentration of chitosan increased, the morphology of the deposited fibers on the collector changed from spherical beads to an interconnected fibrous system. Further, the addition of now in pure chitosan electrospun fiber mats (Jayakumar, Prabaharan, Nair, Tokura, Tamura \& Selvamurugan, 2010; Jayakumar, Prabaharan, Sudheesh Kumar, Nair \& 
95 The electrospinning technique is very versatile and a wide range of parameters can play

96 an important role in obtaining the desired nanofiber size and microstructure. These

97 parameters include solution viscosity, voltage, feed rate, solution conductivity,

98 capillary-to-collector distance and capillary tube size (Bhardwaj \& Kundu; Sill \& von

99 Recum, 2008). In this work, a systematic study has been performed in order to solve the

100 aforementioned main problems. By controlling solution parameters a stable process has

101 been achieved allowing obtaining large fiber mats with tailored fiber dimension.

102 Further, the effect of the main processing parameters such as solvent concentration,

103 flow rate, applied voltage, feed rate and inner needle diameter on the chitosan fiber

104 characteristics and sample morphology has been studied in order to set the ground for a

105 systematic and reproducible way to obtain chitosan nanofiber samples for specific 106 applications.

107

108

\section{Experimental}

109

110

111

112

113

114

115

116

117

118

119

\section{6}

Materials

Chitosan practical grade polymer was purchased from Sigma-Aldrich with $\geq 75 \%$ degree of D-acetylation. Dichloromethane (DCM) and Trifluoroacetic acid (TFA, $99 \%$ ReagentPlus) were purchased from Sigma-Aldrich (Table 1). All materials were used as received from the provider.

Table 1 - (Budavari, 1996). 
The polymer was dissolved in a TFA/DCM solution with different TFA/DCM volume ratios for a $7 \%$ (weight) of chitosan. The solution were prepared under a constant and vigorous magnetic stirring (JPSelecta, Agimatic-E) at room temperature until complete dissolution of the chitosan. The viscosity of the prepared solutions was measured in a Viscostar Plus set-up from Fungilab. The variation of the viscosity of the polymer solution with varying TFA/DCM ratio is represented in figure 1.

\section{Electrospinning}

130 The polymer solution was placed in a commercial plastic syringe fitted with a steel needle. The inner diameter of the needle was $0.5,1.0$ and $1.7 \mathrm{~mm}$ for different experiments. Electrospinning was conducted by applying a voltage ranging from 20 and (Syringepump) fed the polymer solution into the tip at a rate between 1 and $8 \mathrm{ml} . \mathrm{h}^{-1}$. different distances from 50 to $200 \mathrm{~mm}$ from the needle tip.

\section{Characterization}

Electrospun fibers were coated with a thin gold layer using a sputter coater from Polaron (SC502) and the morphology of the membranes were observed by scanning electron microscopy (SEM, JSM-6300 from JEOL) at an accelerating voltage of $15 \mathrm{kV}$.

142 The fiber diameter distribution was calculated over 50 fibers with the Image J software $(\mathrm{J}, 2011)$ from the SEM images obtained at a magnification of $3500 \mathrm{x}$. 
The degree of deacetylation was determined by nuclear magnetic ressonance (NMR) according to the procedure described in (Fernandez-Megia, Novoa-Carballal, Quiñoá \& Riguera, 2005). Five milligram of chitosan after and before electrospun were added to a $5 \mathrm{~mm}$ NMR tube containing $0.5 \mathrm{~mL}$ of $2 \%$ deutorium chloride (DCl, from Fluka) solution in deuterated water $\left(\mathrm{D}_{2} \mathrm{O}, \mathrm{Mw}=20,02\right.$, ACROS Organics $)$ and heated at $70{ }^{\circ} \mathrm{C}$ for $1 \mathrm{~h}$ in order to speed up the dissolution. The results for the ${ }^{1} \mathrm{NMR}$ were collected in a Varian Unity Plus 300 at $70{ }^{\circ} \mathrm{C}$.

\section{Results and Discussion}

Several parameters affect the fiber morphology and size distribution of the polymer electrospun fibers. Among the most important ones are those corresponding to the initial polymer solution: parameters related to the solvent used (dielectric constant, volatility, boiling point and others), the solution concentration (that controls the viscosity) and the molecular weight of the polymer (that allows polymer entanglement). The main parameters that control the jet formation and solvent evaporation rate are the feed rate through the needle and needle diameter, traveling distance from the needle to the collector, temperature and electric field (Ribeiro, Sencadas, Ribelles \& LancerosMéndez, 2010; S. Ramakrishna, K. Fujihara, W. E. Teo, T. C. Lim \& Ma, 2005; Teo \& Ramakrishna, 2006).

TFA is a strong acid that can dissolve the polymer through the formation of salts that destroy the strong interactions that exist between the molecules of chitosan (Ohkawa, Cha, Kim, Nishida \& Yamamoto, 2004). The salt formation occurs between the TFA and the amino groups along the chitosan chain after the following sequential steps: first, 
169 protonation of amine groups $\left(-\mathrm{NH}_{2}\right)$ along the chain of chitosan; second, ionic 170 interaction between protonated amino groups $\left(-\mathrm{NH}_{3}\right)$ and then formation of 171 trifluoroacetate anions. In this configuration, the salts are soluble in an aqueous media.

172 In this work, solutions of chitosan in TFA/DCM with different relative concentrations 173 were used in order to taylor viscosity and conductivity of the solvent. These parameters 174 have strong influence in the electrospinning process and therefore in the final fiber morphology. The SEM images for the electrospun samples obtained from a solution of chitosan with different TFA/DCM volume ratios and fixed traveling distance of 150 $\mathrm{mm}$, needle diameter of $0.5 \mathrm{~mm}$, flow rate of $2 \mathrm{ml}^{-1}$ and a voltage of $25 \mathrm{kV}$ are presented in Figure 2.

179 Schiffman et al. reported that electropinning of chitosan in pure TFA solvent was viable for lower chitosan concentrations $(2.7 \%(\mathrm{w} / \mathrm{v}))$ (Schiffman \& Schauer, 2006). In the present work, it was dificult to stabilize the electrospinning process for TFA/DCM concentrations rich in TFA solvent (higher than $80 \%$ TFA) in the solution due to the presence of sparks that frequently appeared during the fiber processing, even for small applied electric fileds, therefore hindering the electrospinning process. An increase of TFA in the solution increases the viscosity (figure 1) and the conductivity of the medium, which are at the origin of the sparks. Electrospinning involves stretching of the solution caused by the repulsion of the charges at its surface. If the conductivity of the solution is increased, more charges can be carried out by the electrospinning jet. The free amines trifluoroacetate anions formed during the chitosan dissolution in TFA increase the conductivity of the solution (Dannhauser \& Cole, 1952) and, therefore, the critical voltage for electrospinning to occur is reduced. polymer was extremely dificult and during the electrospinning process some drops 
falled from the needle due to the lower viscosity of the solution, These drops complety dissolved the formed fiber and destroyed the homogenety of the electrospun mats. It was also observed that the average fiber diameter of the electrospun mats decreased with increasing DCM content in the solvent mixture. On the other hand, a larger fiber size distribution was observed with increasing TFA content in the solution a (Figure 3).

Figure 3 was obtained from histograms analogous to those shown in Figure 2 ( the ones corresponding to Figures $2 \mathrm{c}$ and $2 \mathrm{~d}$ are included in Figure 3), from which the mean value was calculated. The bars in Figure 3 indicate the average and the standard deviation of the fiber diameters.

The influence of the inner diameter of the needle in the average size of the electrospun fibers was characterized. For the samples processed with a needle with an inner diameter of $0.5 \mathrm{~mm}$, the presence of very thin fibers with diameters $\sim 250 \mathrm{~nm}$ was observed. This fact can be attributed to the lower DCM solvent evaporation temperature (Figure 4). On the other hand, the samples obtained with a higher needle diameter show a more uniform fiber size distribution. It was also noted that all samples were free of beads, indicating that the tested chitosan electrospinnig conditions provide sufficient chain entanglement for fiber formation.

The presence of small particles (Figure $4 \mathrm{~b}$ ) on the surface of the electrospun fibers has been explained by Zhang et al. as a consequence of the presence of salts (Zhang, Yuan, Wu, Han \& Sheng, 2005) originated by the chitosan dissolution in the TFA acid, as explained above. These salts are commonly observed for higher polymer concentrations and higher TFA content in the chitosan-TFA/DCM solvent solution.

The average size of the fibers for the different needle inner diameters was calculated and the results show a slight increase of the average fiber diameter from $\sim 360$ to 410 $\mathrm{nm}$ with increasing inner needle diameter (figure 5). 
219 The fiber diameter distribution along the sample is quite similar for all the electrospun samples, being therefore independent of the needle inner diameter. Literature shows contradictory results in this point. Macossay et al. found no influence of the needle diameter on the average fiber diameter of poly(methyl methacrylate) electrospun fibers (Macossay, Marruffo, Rincon, Eubanks \& Kuang, 2007), while Katti et al. and Ribeiro et al. reported that the fiber diameter decreases with decreasing needle inner diameter (Katti, Robinson, Ko \& Laurencin, 2004; Ribeiro, Sencadas, Ribelles \& LancerosMéndez, 2010).

A decrease of the inner diameter of the needle causes a reduction of the droplet at the tip and therefore the surface tension of the droplet increases. Then, for a given applied voltage, a larger Coulombic force is required to cause the jet initiation, which results in a decrease of the jet acceleration and, as a consequence, more time is required for the solution to be stretched and elongated before it is collected (S. Ramakrishna, K. Fujihara, W. E. Teo, T. C. Lim \& Ma, 2005).

The influence of the distance between the needle tip to the grounded collector on the fiber average diameter and distribution was also analyzed. It was observed that the fibers with the smallest average diameter, $\sim 260 \mathrm{~nm}$, were obtained for the samples with a $50 \mathrm{~mm}$ distance between needle tip and collector and that the mean fiber diameter increases by increasing the distance between the needle tip and the collector. A maximum average fiber diameter of $\sim 500 \mathrm{~nm}$ was obtained for a traveling distance of $200 \mathrm{~mm}$ (Figure 6).

It was also observed that the mean diameter fiber distribution increases with increasing the distance between needle tip and sample collector. The presence of sub-structures of smaller fibers between the smooth large fibers (Figure 7) suggests the formation of a secondary jet during the main electrospinning process due to the high solution viscosity 
(Figure 1). Ding et al. (Ding \& et al., 2006) pointed out that this fact is related to certain processing conditions such as high voltage, low relative humidity and fast phase separation of polymer and solvent during the flight between the needle and the collector. justified these structures as a consequence of the formations and ejection of smaller jets from the surface of the primary jets, which is comparable to the ejection of the initial jet from the surface of a charged droplet. It was proposed that the elongation of the jet and evaporation of the solvent modifies the shape and the charge density of the jet during the traveling between the tip and the collector. Thus, the balance between the electrical forces and surface tension can change, giving rise to instabilities in the shape of the jet. Such instabilities can decrease the local charge per unit surface area by ejecting a smaller jet from the surface of the primary jet or by splitting apart into two smaller jets. In this work a blend of two solvents with different boiling points (Table 1) has been used and the observed sub-structures of smaller fibers can be related to the fast that crystallize later when the TFA solvent evaporates. This phenomenon was also observed in Figures 2 and 3, when the effect of TFA/DCM solvent ratio on fiber diameter and mat morphology was presented.

It is pointed out that an increase of the distance between the tip and the collector often results in a decrease of the fiber diameter (S. Ramakrishna, K. Fujihara, W. E. Teo, T. C. Lim \& Ma, 2005). However, in the present work it was observed that the diameter of the fibers increases for increasing distance between the needle tip and the grounded collector. This behavior is to be ascribed to the decrease of the electrostatic field 
strength resulting in a decrease of the electrostatic force and therefore on the stretching of the fibers.

Tip to collector distance has a direct influence on the jet flight time and electric field strength: a decrease of the distance shortens flight and solvent evaporation times and increases the electric field strength. A decrease in the tip-collector distance has a similar effect as increasing the voltage (Figure 8).

The changes in the applied electric field have strong influence on the shape of the droplet at the needle tip, its surface charge, dripping rate, velocity of the flowing fluid and hence on the fiber structure and morphology. Similarly, the needle tip to collector distance also determines the time available for fiber drying and the space available for fiber splaying and whipping to take place.

The high voltage will induce the necessary charge distribution on the solution and initiate the electrospinning process when the electrostatic force overcomes the surface tension of the solution (S. Ramakrishna, K. Fujihara, W. E. Teo, T. C. Lim \& Ma, 2005). For higher electric fields, the jet will accelerate and stretch due to the larger Coulombic forces, which results in a reduction of the fiber average diameter and also promotes faster solvent evaporation to yield drier fibers (S. Ramakrishna, K. Fujihara, W. E. Teo, T. C. Lim \& Ma, 2005). Finally, the influence of the feed rate on the average fiber distribution was characterized (Figure 9). A minimum value of solution volume suspended at the end of the needle should be maintained in order to form a stable Taylor cone (Teo \& Ramakrishna, 2006). The feed rate determines the amount of solution available for the electrospining process. Typically, when the feed rate increases, a corresponding increase of the fiber diameter is observed, as observed i.e. for poly(vinylidene fluoride) (Ribeiro, Sencadas, Ribelles \& Lanceros-Méndez, 2010) and for poly(L-lactide acid) (Clarisse \& et al., 2011). In 
chitosan such behavior was not found and the fiber diameter distribution is quite similar for the different feed rates within the range studied in the present work (Figure 9). It was expected that increasing feed rate will increase the volume of the solution drawn

297 from the needle tip, and consequently the jet would take a longer time to dry. The lower boiling point of the solvents used in this work (Table 1) allows the fast evaporation during the flight time. In this situation, full solvent evaporation has already occurred when the fiber reaches the grounded collector and therefore the feed rate does not have influence on the fiber diameter.

Most of the physical and chemical properties of this biopolymer strongly depend on the degree of deacetylation (DD) (Lavertu et al., 2003). The DD can be calculated from the ${ }^{1} \mathrm{NMR}$ spectra (figure 10) through:

$$
D D(\%)=\frac{H_{1} D}{H_{1} D+\left(\frac{H_{a c}}{3}\right)} \times 100
$$

where $\mathrm{H}_{1} \mathrm{D}$ is the peak corresponding to the $\mathrm{H}_{1}$ proton of the deacetylated monomer (duplet at $\delta=4.858 \mathrm{ppm}$ ) and $\mathrm{H}_{\mathrm{ac}}$ is the peak of the three protons of the acetyl group (singlet at $\delta=1.988 \mathrm{ppm}$ ) (Lavertu et al., 2003). The obtained results show that the commercial chitosan has a DD of $78 \%$, which is similar to the value given by the producer and also similar to the values obtained for the electrospun fibers. It is therefore concluded that the electrospinning process does not affect the degree of deacetylation of the polymer.

\section{Conclusions}


317 Large chitosan mats with uniform fibers of submicron diameters without beads have been prepared from trifluoroacetic acid / dichloromethane mixture solutions by a stable electrospinnig process. It was observed that an increase of the DCM present in the solvent blend solution produces nanofibers with smaller diameters and narrower diameter distribution. Inclusion of DCM within the TFA solutions modifies solution viscosity and electrical characteristics, leading to a stable flow rate and avoiding spark formation. The inner diameter of the needle and the feed rate does not have influence in the chitosan electrospun fiber diameter. On the other hand, it was observed that a decrease of the distance from the needle tip to the grounded collector gives origin to nanofibers with smaller diameters. Finally, an increase of the applied voltage also decreases the nanofibers diameter. The degree of deacetylation of the polymer is not affected by the electrospinning process.

\section{Acknowledgements}

This work is funded by FEDER funds through the "Programa Operacional Factores de Competitividade - COMPETE" and by national funds by FCT- Fundação para a Ciência e a Tecnologia, project references NANO/NMed-SD/0156/2007. V.S. thanks the FCT for the SFRH/BPD/63148/2009 grants. JLGR acknowledge the support of the Spanish Ministry of Science and Innovation through project No. MAT2010-21611-C03-01 (including the FEDER financial support) and Programa Nacional de Internacionalización de la I+D project EUI2008-00126. Funding for research in the field of Regenerative Medicine through the collaboration agreement from the Conselleria de Sanidad (Generalitat Valenciana), and the Instituto de Salud Carlos III (Ministry of Science and Innovation) is also acknowledged. 
342 Thanks are due to the National NMR Network that was purchased within the framework of the National Program for Scientific Re-equipment, contract REDE/1517/RMN/2005 with funds from POCI 2010 (FEDER) and FCT. Also thank to the UPV Microscopy Service for the use of their lab.

\section{References}

Baldrick, P. (2010). The safety of chitosan as a pharmaceutical excipient. Regulatory Toxicology and Pharmacology, 56(3), 290-299.

Beachley, V., \& Wen, X. (2010). Polymer nanofibrous structures: Fabrication, biofunctionalization, and cell interactions. Progress in Polymer Science, 35(7), 868892.

Bhardwaj, N., \& Kundu, S. C. Electrospinning: A fascinating fiber fabrication technique. Biotechnology Advances, 28(3), 325-347.

Budavari, S. (1996). An Encyclopedia of Chemicals, Drugs, and Biologicals. New Jersey: Merck \& Co.

Clarisse, R., \& et al. (2011). Tailoring the morphology and crystallinity of poly(Llactide acid) electrospun membranes. Science and Technology of Advanced Materials, 12(1), 015001.

Dannhauser, W., \& Cole, R. H. (1952). On the Dielectric Constant of Trifluoroacetic Acid1. Journal of the American Chemical Society, 74(23), 6105-6105.

Ding, B., \& et al. (2006). Formation of novel 2D polymer nanowebs via electrospinning. Nanotechnology, 17(15), 3685.

Fernandez-Megia, E., Novoa-Carballal, R., Quiñoá, E., \& Riguera, R. (2005). Optimal 
367 Honarkar, H., \& Barikani, M. (2009). Applications of biopolymers I: chitosan. 368 Monatshefte Fur Chemie, 140(12), 1403-1420.

369 J, I. (2011). Image Processing and Analysis in Java available from 370 http://rsbweb.nih.gov/ij/index.html.

371 Jayakumar, R., Menon, D., Manzoor, K., Nair, S. V., \& Tamura, H. (2010). Biomedical 372 applications of chitin and chitosan based nanomaterials--A short review. Carbohydrate 373 Polymers, 82(2), 227-232. Jayakumar, R., Prabaharan, M., Nair, S. V., \& Tamura, H. Novel chitin and chitosan nanofibers in biomedical applications. Biotechnology Advances, 28(1), 142-150. Jayakumar, R., Prabaharan, M., Nair, S. V., Tokura, S., Tamura, H., \& Selvamurugan, N. (2010). Novel carboxymethyl derivatives of chitin and chitosan materials and their biomedical applications. Progress in Materials Science, 55(7), 675-709. Jayakumar, R., Prabaharan, M., Sudheesh Kumar, P. T., Nair, S. V., \& Tamura, H. (2011). Biomaterials based on chitin and chitosan in wound dressing applications. Biotechnology Advances, 29(3), 322-337.

Katti, D. S., Robinson, K. W., Ko, F. K., \& Laurencin, C. T. (2004). Bioresorbable nanofiber-based systems for wound healing and drug delivery: Optimization of fabrication parameters. Journal of Biomedical Materials Research Part B: Applied Biomaterials, 70B(2), 286-296.

Krajewska, B. (2005). Membrane-based processes performed with use of chitin/chitosan materials. Separation and Purification Technology, 41(3), 305-312.

Lavertu, M., Xia, Z., Serreqi, A. N., Berrada, M., Rodrigues, A., Wang, D., Buschmann, 389 M. D., \& Gupta, A. (2003). A validated 1H NMR method for the determination of the degree of deacetylation of chitosan. Journal of Pharmaceutical and Biomedical Analysis, 32(6), 1149-1158. 
Macossay, J., Marruffo, A., Rincon, R., Eubanks, T., \& Kuang, A. (2007). Effect of needle diameter on nanofiber diameter and thermal properties of electrospun poly(methyl methacrylate). Polymers for Advanced Technologies, 18(3), 180-183.

Malafaya, P. B., Silva, G. A., \& Reis, R. L. (2007). Natural-origin polymers as carriers and scaffolds for biomolecules and cell delivery in tissue engineering applications. Advanced Drug Delivery Reviews, 59(4-5), 207-233.

Ohkawa, K., Cha, D., Kim, H., Nishida, A., \& Yamamoto, H. (2004). Electrospinning of Chitosan. Macromolecular Rapid Communications, 25(18), 1600-1605.

Pillai, C. K. S., Paul, W., \& Sharma, C. P. (2009). Chitin and chitosan polymers: Chemistry, solubility and fiber formation. Progress in Polymer Science, 34(7), 641-678. Ravi Kumar, M. N. V. (2000). A review of chitin and chitosan applications. Reactive and Functional Polymers, 46(1), 1-27.

Ribeiro, C., Sencadas, V., Ribelles, J. L. G., \& Lanceros-Méndez, S. (2010). Influence of Processing Conditions on Polymorphism and Nanofiber Morphology of Electroactive Poly(vinylidene fluoride) Electrospun Membranes. Soft Materials, 8(3), 274 - 287.

S. Ramakrishna, K. Fujihara, W. E. Teo, T. C. Lim, \& Ma, Z. (2005). Introduction to electrospinning and nanofibers. Singapure: World Scientific.

Schiffman, J. D., \& Schauer, C. L. (2006). Cross-Linking Chitosan Nanofibers. Biomacromolecules, 8(2), 594-601.

Schiffman, J. D., \& Schauer, C. L. (2007). One-Step Electrospinning of Cross-Linked Chitosan Fibers. Biomacromolecules, 8(9), 2665-2667.

Sill, T. J., \& von Recum, H. A. (2008). Electrospinning: Applications in drug delivery and tissue engineering. Biomaterials, 29(13), 1989-2006.

Teo, W. E., \& Ramakrishna, S. (2006). A review on electrospinning design and nanofibre assemblies. Nanotechnology, 17(14), R89. 
417 Yaghobi, N., \& Hormozi, F. (2010). Multistage deacetylation of chitin: Kinetics study.

418 Carbohydrate Polymers, 81(4), 892-896.

419 Zhang, C., Yuan, X., Wu, L., Han, Y., \& Sheng, J. (2005). Study on morphology of 420 electrospun poly(vinyl alcohol) mats. European Polymer Journal, 41(3), 423-432.

421 
422 Figure 1 - Variation of the viscosity of the polymer solution with varying TFA/DCM 423 ratio. The line is just a guide for the eyes.

Figure 2 - Morphology of the chitosan mats for the samples obtained with a $7 \%(\mathrm{w} / \mathrm{v})$ polymer solution at a traveling distance of $150 \mathrm{~mm}$, needle diameter of $0.5 \mathrm{~mm}$, flow rate of $2 \mathrm{ml}^{-1}$ and a voltage of $25 \mathrm{kV}$ : a) 80:20 and b) 60:40 TFA/DCM (v/v) solution; c) and d) represent the fiber diameter histograms of the corresponding figures.

Figure 3 - Influence of the TFA/DCM volume ratio in the distribution of fiber diameters in the electrospun mats obtained for a $7 \%(\mathrm{w} / \mathrm{v})$ chitosan solution at a fixed traveling distance of $150 \mathrm{~mm}$, needle diameter of $0.5 \mathrm{~mm}$, flow rate of $2 \mathrm{ml}^{-1} \mathrm{~h}^{-1}$ and a voltage of $25 \mathrm{kV}$.

434

Figure 4 - Morphology of the chitosan mats for the samples obtained for a $7 \%(\mathrm{w} / \mathrm{v})$ polymer solution, a 70/30 TFA/DCM solvent solution, a traveling distance of $150 \mathrm{~mm}$, flow rate of $2 \mathrm{ml}^{-1}$ and a voltage of $25 \mathrm{kV}$ for needle inner diameters of a) $0.5 \mathrm{~mm}$ and figures.

Figure 5 - Influence of the inner diameter of the needle in the average fiber diameter and distribution for the electrospun mats obtained for a $7 \%(\mathrm{w} / \mathrm{v})$ chitosan solution, a $70 / 30 \mathrm{TFA} / \mathrm{DCM}$ solvent solution, a traveling distance of $150 \mathrm{~mm}$, flow rate of $2 \mathrm{ml} . \mathrm{h}^{-1}$ and a voltage of $25 \mathrm{kV}$.

Figure 6 - Influence of the traveling distance on the fiber average diameter and distribution in the electrospun mats obtained for a $7 \%(\mathrm{w} / \mathrm{v})$ chitosan solution, a 70/30 TFA/DCM solvent solution, a needle inner diameter of $0.5 \mathrm{~mm}$, a flow rate of $2 \mathrm{ml}^{-\mathrm{h}^{-1}}$ and a voltage of $25 \mathrm{kV}$. 
454 Figure 7 - Morphology of the chitosan mats for the samples obtained for a $7 \%(w / v)$ 455 polymer solution, a 70/30 TFA/DCM solvent solution and a needle diameter of $0.5 \mathrm{~mm}$, 456 at a traveling distance of $150 \mathrm{~mm}$, flow rate of $2 \mathrm{ml}^{-1}$ a voltage of $25 \mathrm{kV}$ and a 457 distance between the needle tip to the sample collector of a) $50 \mathrm{~mm}$ and b) $200 \mathrm{~mm}$.

460 Figure 8 - Influence of the applied voltage on fiber average diameter and distribution in 461 the electrospun mats obtained for a $7 \%(\mathrm{w} / \mathrm{v})$ chitosan solution, a 70/30 TFA/DCM solvent solution, needle inner diameter of $1.7 \mathrm{~mm}$, flow rate of $2 \mathrm{ml}^{-1}$ and a traveling distance of $15 \mathrm{~cm}$.

464

465

Figure 9 - Influence of the feed rate on the average fiber diameter and distribution of 466 the electrospun mats obtained for a $7 \%(\mathrm{w} / \mathrm{v})$ chitosan solution, a 70/30 TFA/DCM solvent solution, needle inner diameter of $0.5 \mathrm{~mm}$, flow rate of $2 \mathrm{ml}^{-1}$, a traveling distance of $15 \mathrm{~cm}$ and a $25 \mathrm{kV}$ applied voltage.

469

470

Figure $10-{ }^{1} \mathrm{H}-\mathrm{NMR}$ spectra of electrospun chitosan nanofibers at $70{ }^{\circ} \mathrm{C}$.

471

472 Table 1 - Properties of the solvents used in the present work [17]. 
476 Table 1

\begin{tabular}{|c|c|c|c|c|c|}
\hline Solvent & $\begin{array}{c}\text { Melting } \\
\text { Point }\end{array}$ & $\begin{array}{c}\text { Boiling } \\
\text { Point }\end{array}$ & Density & $\begin{array}{c}\text { Dipole } \\
\text { Moment }\end{array}$ & $\begin{array}{c}\text { Dielectric } \\
\text { Constant }\end{array}$ \\
\hline & ${ }^{\circ} \mathrm{C}$ & ${ }^{\circ} \mathrm{C}$ & $\mathrm{g} . \mathrm{cm}^{-3}$ & Debye & \\
\hline TFA & -15.2 & 73.0 & 1.535 & 2.28 & 8.42 \\
\hline DCM & -95.1 & 40.0 & 1.327 & 1.60 & 8.93 \\
\hline
\end{tabular}

477

478

479 
$480 \quad$ Figure 1

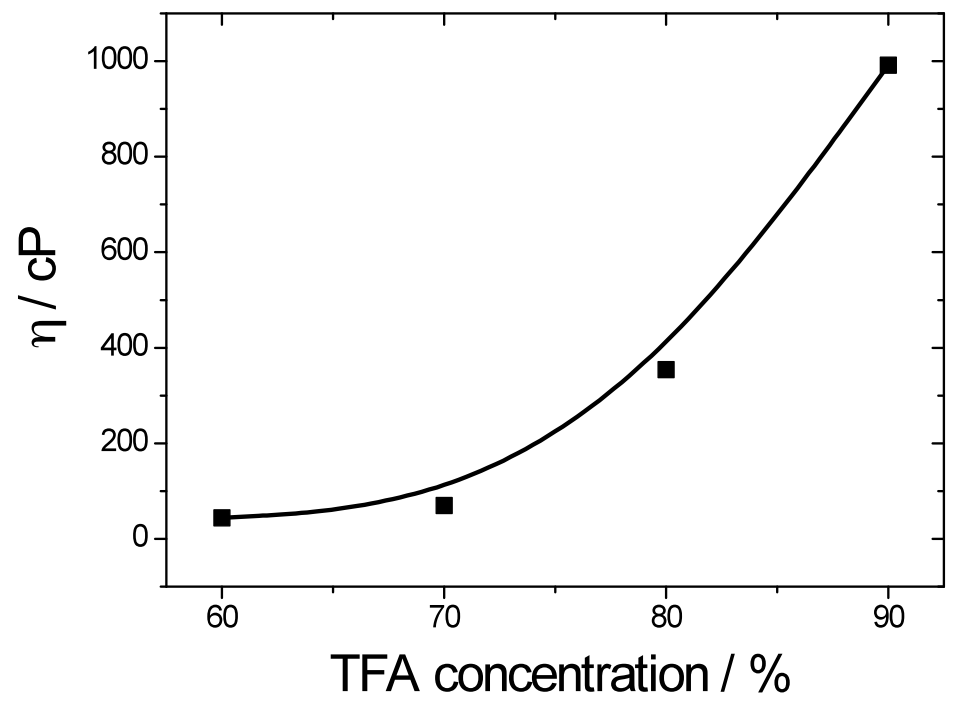

481

482 
$483 \quad$ Figure 2

484
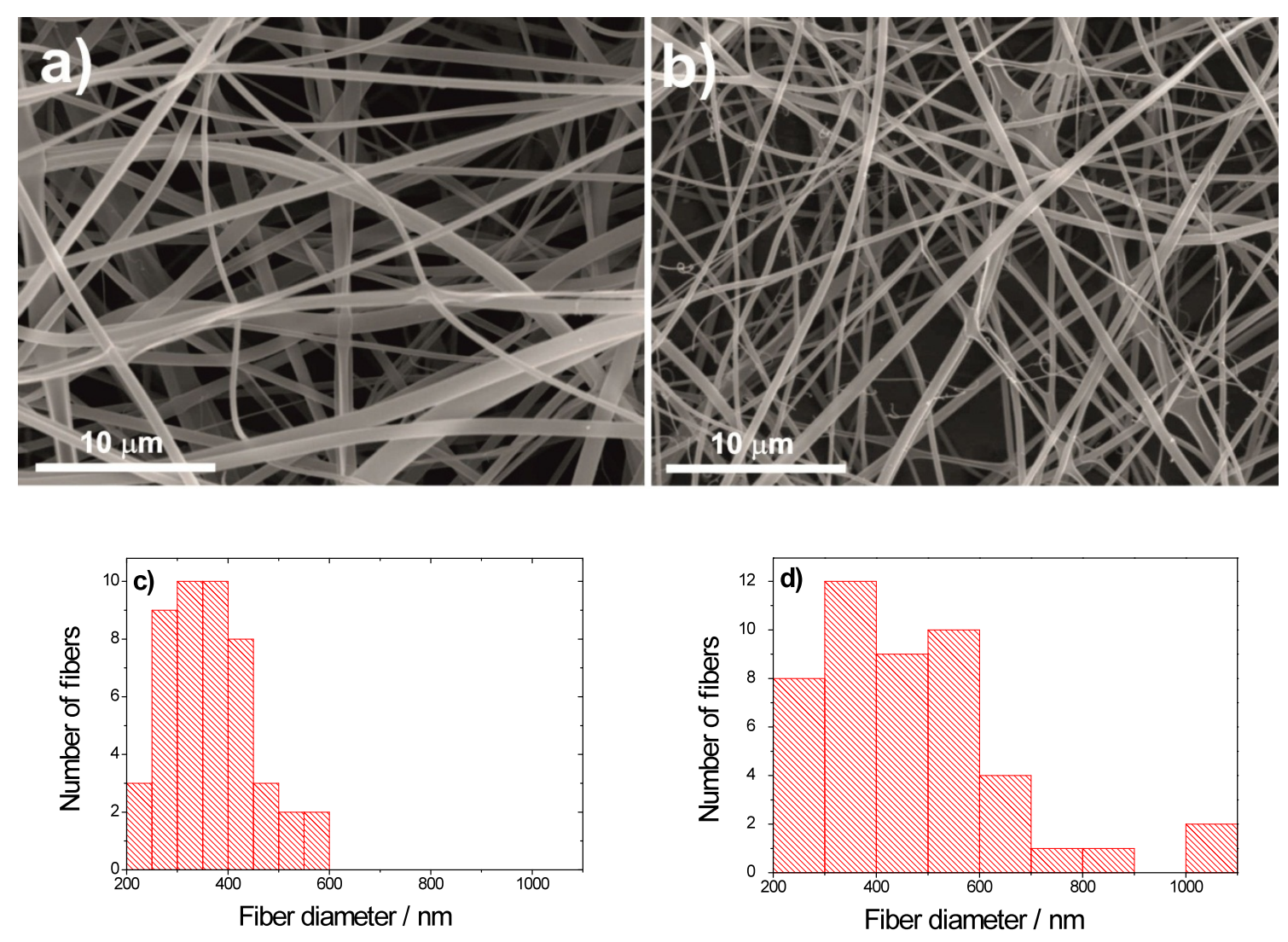

485 
$487 \quad$ Figure 3

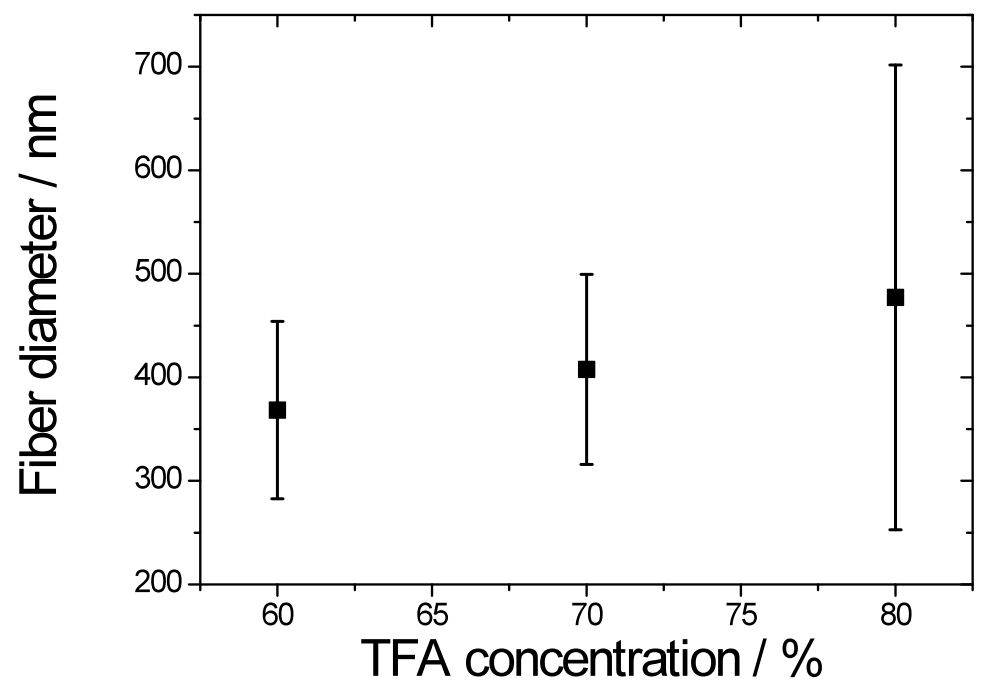

488

489 
$490 \quad$ Figure 4
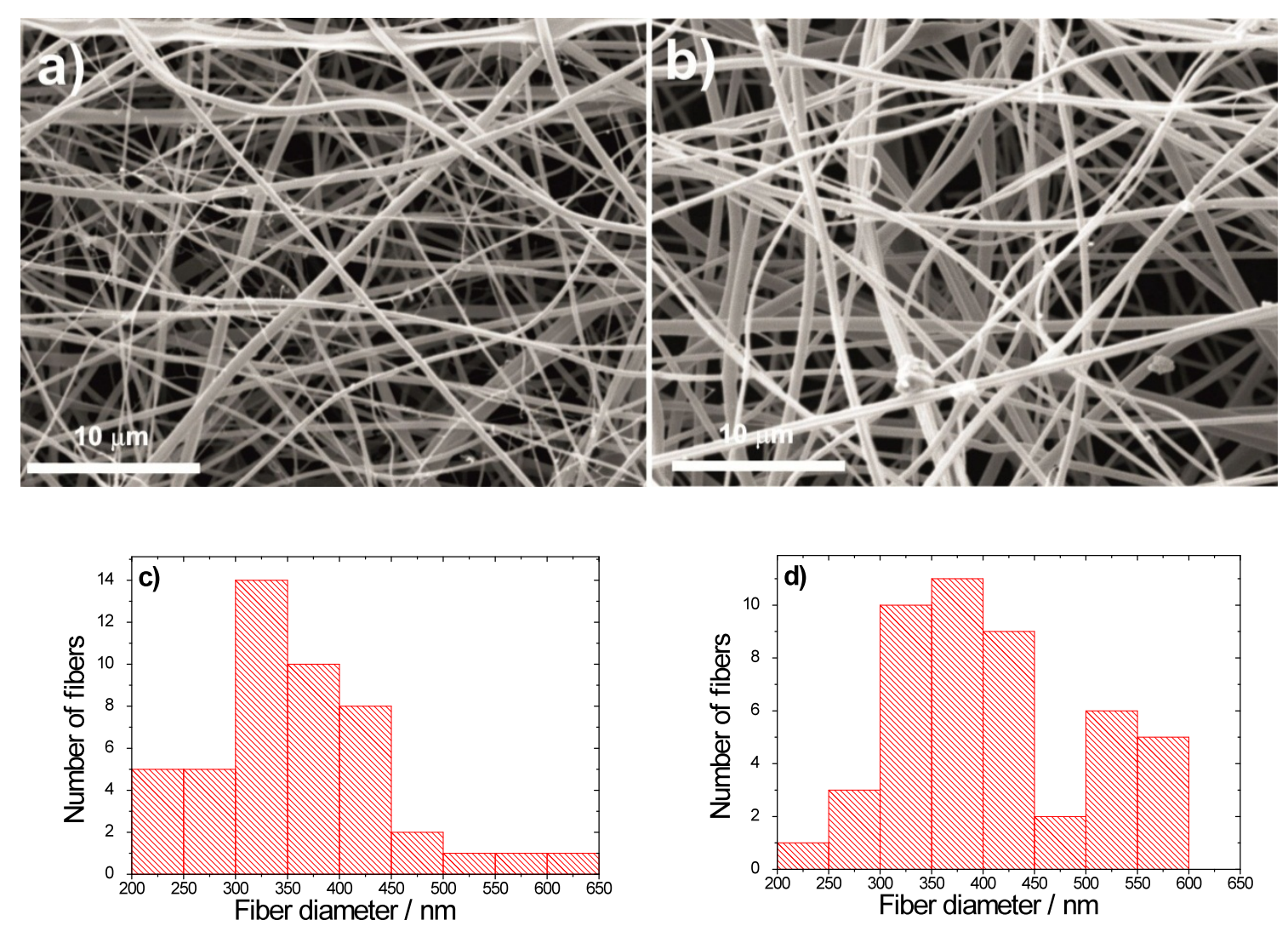

491

492 
$493 \quad$ Figure 5

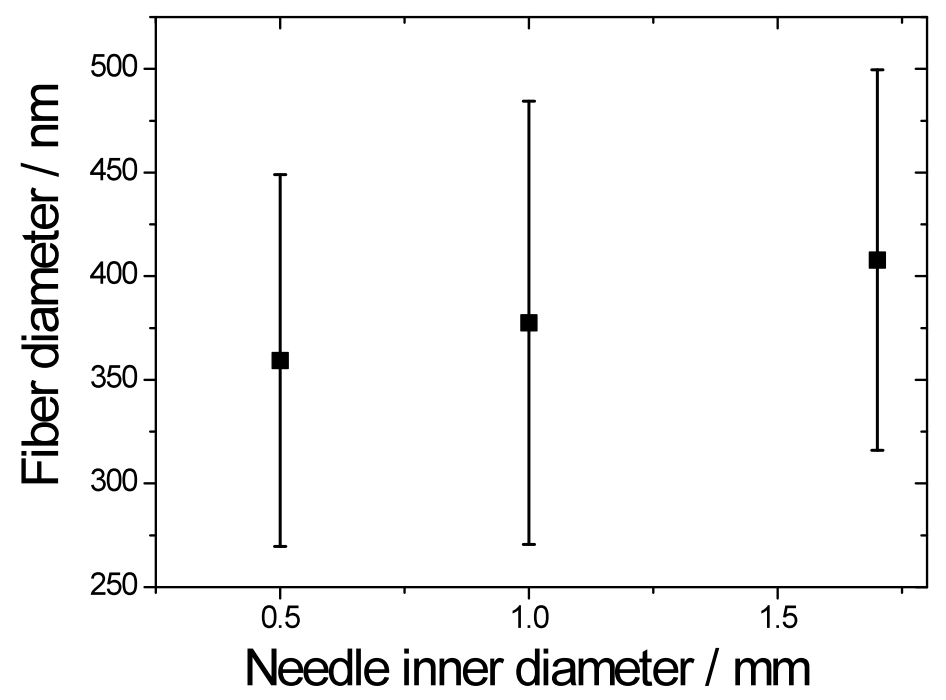

494

495 
$496 \quad$ Figure 6

497

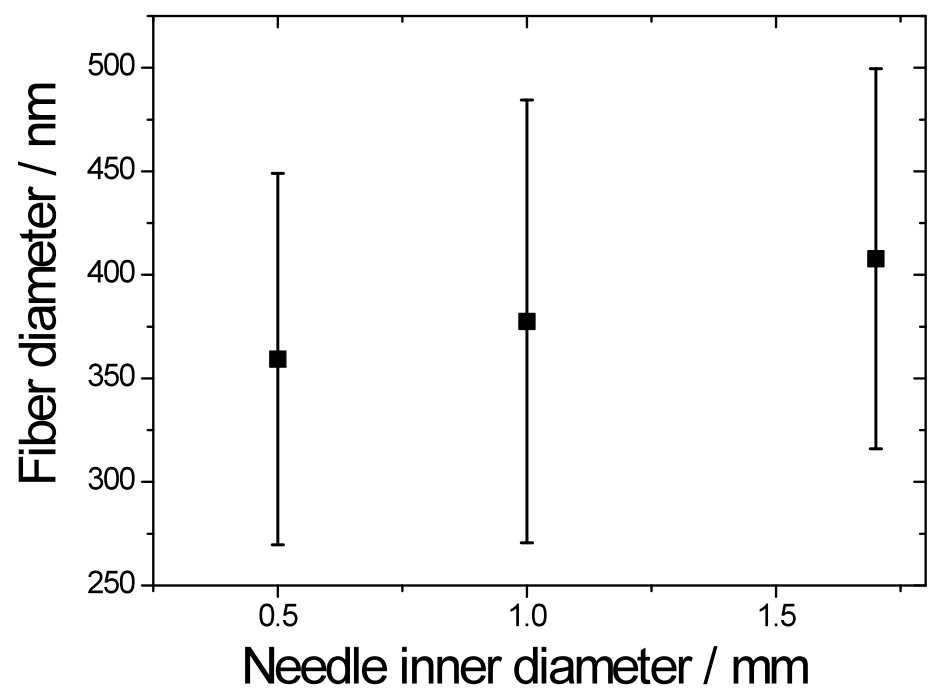

498

499 
$500 \quad$ Figure 7

501

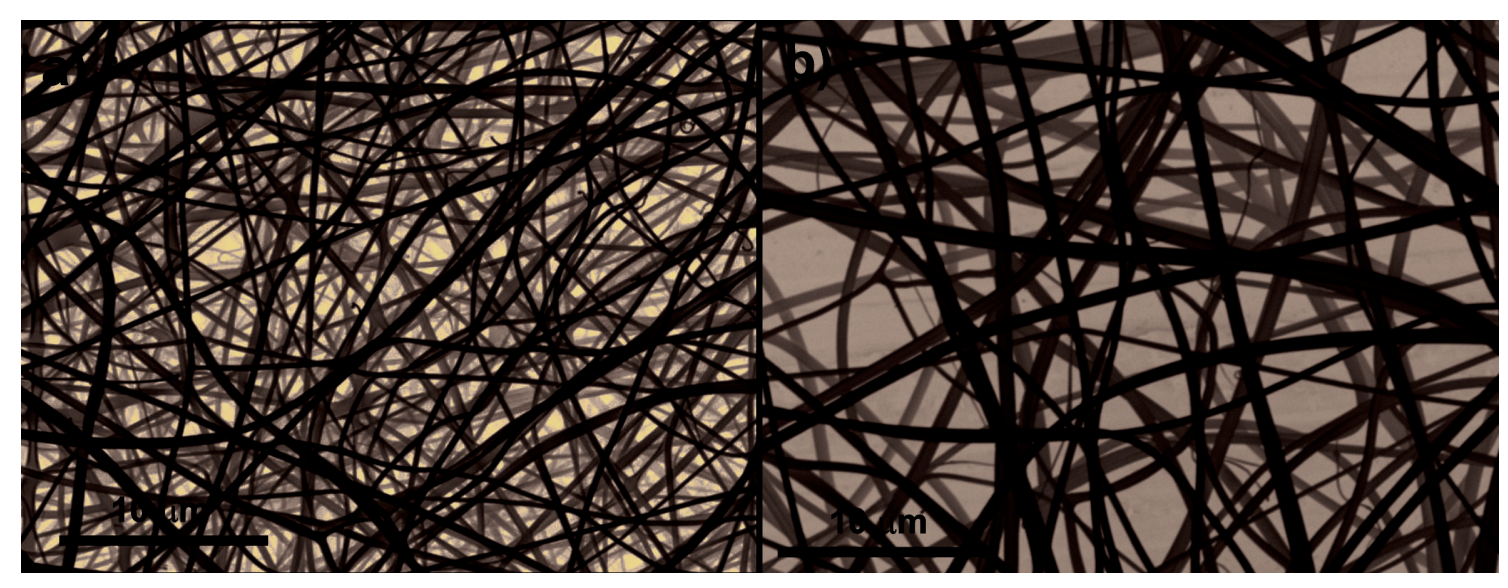

502

503 
$504 \quad$ Figure 8

505

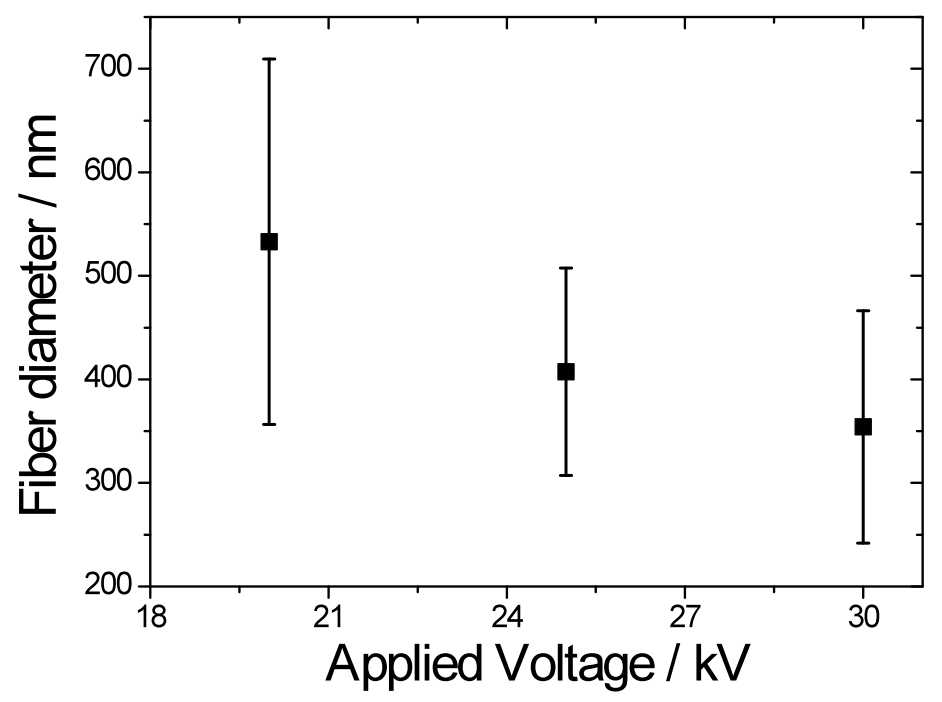

506

507 
Figure 9

509

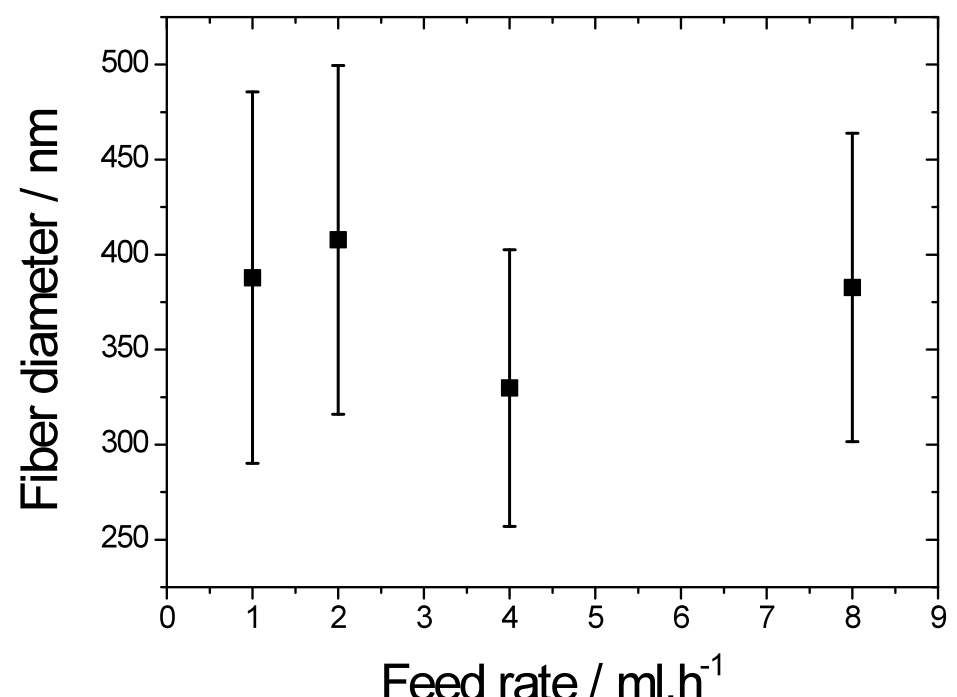

510

511 
512 Figure 10

513

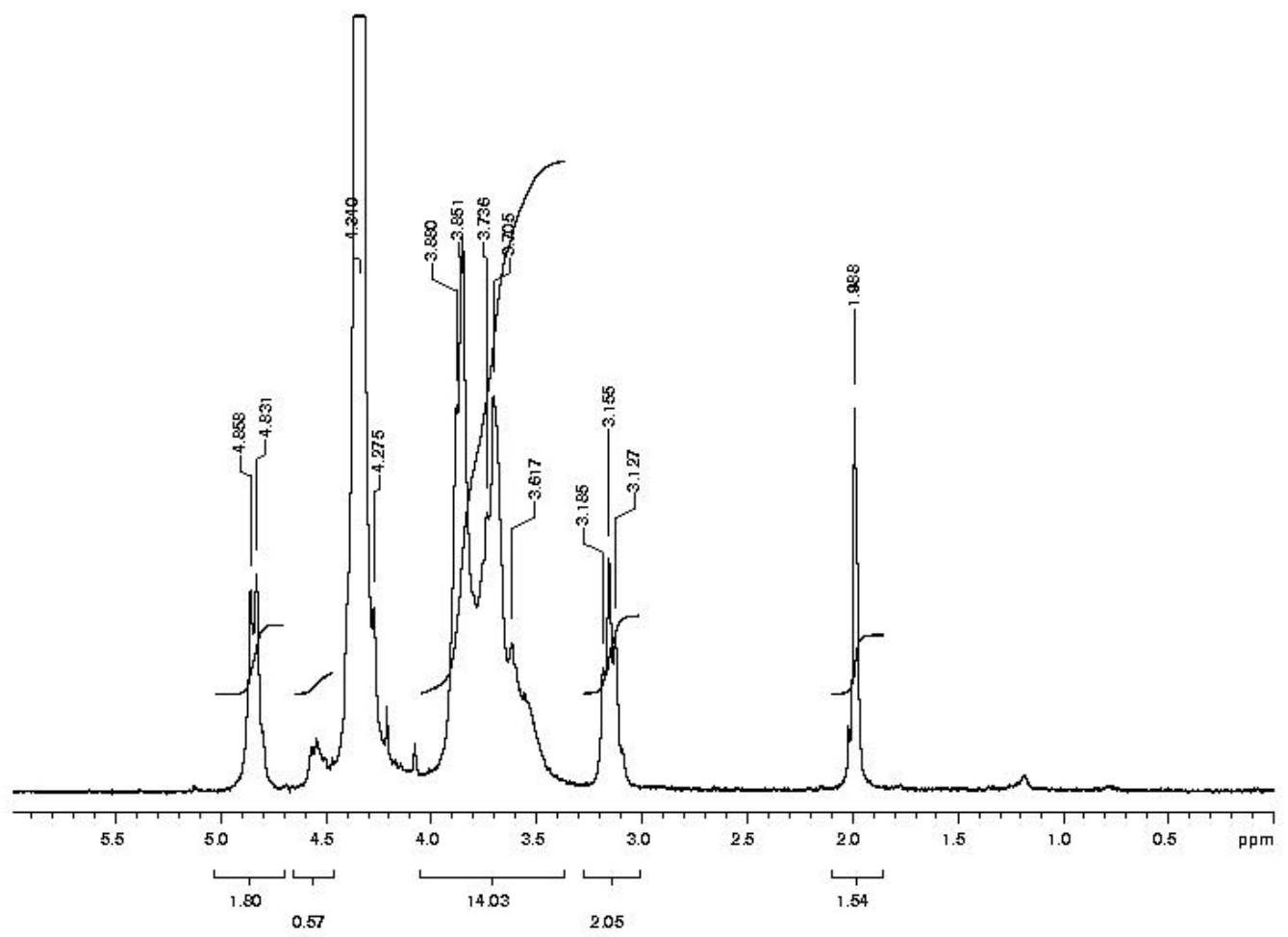

